J. Lake Sci. (湖泊科学), $2006, \mathbf{1 8}(3): 293-298$

http:// www. jlakes. org. E-mail: jlakes@ niglas. ac.cn

(c) 2006 by Journal of Lake Sciences

\title{
中华线螯蟹病原鳗利斯顿氏菌生物学特性”
}

\author{
陈翠珍, 房 海, 张晓君, 葛慕湘, 王秀云, 靳晓敏
}

(河北科技师范学院动物科学系, 秦皇岛 066600)

摘 要: 从一起自然发病的中华线鳌蟹 (Eriocheir sinensis H. Milne-Edwards) 肝胰腺中,分离做纯培养的 6 株( HQ010516A-1 至 HQ010516A-6) 鳗利斯顿氏菌 ( Listonella anguillarum), 进行了形态特征、主要理化特性等方面的检验. 同时择代表菌 株(HQ010516A-1) 测定了 16S rRNA 基因序列、构建了系统发育树. 另外, 以代表菌株(HQ010516A-1) 对健康蟹进行了人 工感染试验,结果表明了该菌株在所检病例的病原学意义;用 37 种抗菌类药物对 3 株菌所做的药敏试验,结果均表现对 头狍噻肜等 29 种药物呈现敏感或高度敏感 (抑菌圈直径在 16-40 mm)、对头狍唑啉等 3 种药物呈低度敏感 (抑菌圈直径 在 $10-12 \mathrm{~mm}$ )、对青霉素 $\mathrm{G}$ 等 5 种药物耐药 (无抑菌圈形成).

关键词:中华线鳌蟹;鳗利斯顿氏菌;生物学特性

\section{Biological characterization of Listonella anguillarum isolated from Crab (Eriocheir sinensis L. )}

\author{
CHEN Cuizhen, FANG Hai,ZHANG Xiaojun, GE Muxiang, WANG Xiuyun \& JIN Xiaomin \\ (Department of Animal Science, Hebei Normal University of Science and Technology, Qinhuangdao 066600, P. R. \\ China)
}

\begin{abstract}
Examination of L. anguillarum ( 6 strains:HQ010516A-1 to HQ010516A-6) which were isolated from liver of naturally infected crab (Eriocheir sinensis L.) was conducted including the morphological characteristics, main physiological and biochemical characteristics, 16S rRNA gene sequence was detected and phylogenetic analysis was constructed. In the same time, artificial infection experiment of representative strain (HQ010516A-1) to healthy crab was conducted, the results showed corresponding pathogenicity of the strain. The tests of antibiotic sensitivity of 3 strains to used 37 antimicrobial agents showed that all strains were sensitive or high sensitive to 29 agents including cefotaxime et al. ( Diameter of the zones of inhibition was $16-40 \mathrm{~mm}$ ), were slight sensitive to 3 agents including cefazolin et al. (Diameter of the zones of inhibition was $10-12 \mathrm{~mm}$ ), were resistant to 5 agents including penicillin $\mathrm{G}$ et al. (There was no zones of inhibition).
\end{abstract}

Keywords : Crab (Eriocheir sinensis L. ) ; Listonella anguillarum; Biological characterization

利斯顿氏菌属 (Listonella MacDonell and Colwell 1986) 的鳗利斯顿氏菌 (Listonella anguillarum), 即原弧 菌属 (Vibrio Pacini 1954) 的鳗弧菌 (V. anguillarum). 已知该菌为重要的水产动物病原菌,能引起多种鱼类 如大鳞大麻哈鱼、大菱鲆、香鱼、鳗鲡、太平洋鲑、红点鲑、鲇鱼、鲽鱼及贝类、甲壳类等发生相应的“弧菌病 (Vibriosis)”, 常能给水产养殖生产造成严重危害 ${ }^{[1,2]}$.

2001 年 5 月初, 河北某水产养殖场所养殖的一年生中华线鳌蟹发生病害,病蟹主要表现为行动迟缓、 少食至不食; 至 5 月 16 日检查时,随机捞取一定数量检查其平均发病率 $35 \%$ 、死亡率 $22 \%$. 经做病原学检 验, 表明其为利斯顿氏菌属的鳗利斯顿氏菌、气单胞菌属( Aeromonas Kluyver and van Niel) 的维氏气单胞菌 (A. veronii)、柠檬酸杆菌属 (Citrobacter werkman and Gillen) 的弗氏柠檬酸杆菌 (C. freundii) 的混合感染. 鉴 于鳗利斯顿氏菌在水产养殖动物中的重要病原学意义,为丰富该菌的一些生物学性状内容, 我们对所分离 做纯培养的 6 株菌进行了在形态特征、主要理化性状及系统发育学、病原学意义、对抗菌类药物敏感性等方

*2005-06-21 收稿;2005-10 - 收修改稿; 陈翠珍,女,1955 年生,教授. E-mail: chencuizhen@ 163.com. 
面的研究, 旨在为该菌的有效检验提供一定的参考.

\section{1 材料与方法}

\section{1 细菌分离与纯培养}

随机取病蟹及刚刚病死蟹各 6 只共 12 只, 剥开背甲观察均见体腔内有不等量积水, 鳃呈暗灰色, 肝胰 腺呈浅黄色等不同程度的病理变化. 取经检查后的病蟹及病死蟹各 3 只共 6 只, 分别以其肝胰腺为材料, 先分别做涂片经革兰氏染色镜检细菌; 再分别做划线接种于普通营养琼脂、血液营养琼脂(含 $7 \%$ 家免脱纤 血液的营养琼脂) 培养基, $28^{\circ} \mathrm{C}$ 培养 $24-48 \mathrm{~h}$ 做细菌分离; 待分离细菌后, 在先检查菌落种类、数量及分布情 况的基础上, 选取每种细菌菌落的一定数量, 分别移接于普通营养琼脂培养基斜面做成纯培养 $\left(28^{\circ} \mathrm{C}\right.$ 培养 $24 \mathrm{~h}$ ) 供鉴定用.

\section{2 纯培养菌的主要理化特性测定及系统发育学分析}

1.2 .1 形态特征检查 取上述纯培养菌, 分别移接于普通营养琼脂斜面, 置 $28^{\circ} \mathrm{C}$ 条件下培养 $18 \mathrm{~h}$ 后制备成 涂片标本, 经革兰氏染色镜检细菌形态.

1.2 .2 在不同培养基上的生长及菌落特征检查 取上述纯培养菌, 分别划线接种于普通营养琼脂、血液琼 脂( 分别为含 7\% 家免脱纤血及含 7\% 绵羊脱纤血的营养琼脂)、沙门氏菌志贺氏菌琼脂 (SS 琼脂) 、疤疮病 琼脂 (FA)、胰胨大豆胨琼脂 (TSA) 、木糖赖氨酸去氧胆酸盐琼脂 (XLD)、庆大霉素琼脂、硫代硫酸钠柠檬酸 钠蔗糖琼脂 (TCBS)、麦康凯琼脂、伊红美蓝琼脂、Rimler-Shotts 培养基( RS) 及 2216E 海水培养基(2216E) 等 12 种不同培养基平板, 置 $28^{\circ} \mathrm{C}$ 培养 $24 \mathrm{~h}$ 和 $48 \mathrm{~h}$, 分别检查生长情况及菌落特征.

1.2 .3 培养温度对细菌生长的影响 取上述纯培养菌, 分别做涂布接种于普通营养琼脂斜面各 2 管, 分置 于 $37^{\circ} \mathrm{C}$ 和 $28^{\circ} \mathrm{C}$ 培养 $48 \mathrm{~h}$, 检查细菌生长情况.

1.2 .4 在普通营养肉汤中生长情况检查 取上述纯培养菌, 分别接种于普通营养肉汤(管) 中, 置 $28^{\circ} \mathrm{C}$ 培养 $24 \mathrm{~h}$, 检查液体培养的生长表现.

1.2 .5 理化特性测定 取上述纯培养菌, 分别移接于细菌鉴定的相应培养基进行氧化酶、 $\mathrm{H}_{2} \mathrm{O}_{2}$ 酶、糖(醇和 苷) 类代谢、吲哚、MR 试验、V-P 反应、有机酸盐利用、 $\mathrm{H}_{2} \mathrm{~S}$ 产生、硝酸盐还原等试验, 较系统地测定其相应理 化特性 ${ }^{[3]}$.

\subsubsection{S rRNA 基因序列测定与系统发育学分析}

（1）菌株 DNA 样本的制备：选取上述分离鉴定的 1 个代表菌株接种于 LB 肉汤中 $37^{\circ} \mathrm{C}$ 培养 $16 \mathrm{~h}$,用 上海华舜生物工程有限公司生产的 “小量细菌基因组 DNA 抽提试剂盒( W6511)” 提取 DNA 作为 PCR 模板 DNA.

（2） $16 \mathrm{~S} \mathrm{rRNA}$ 基因序列的 PCR 扩增与测序： $16 \mathrm{~S} \mathrm{rRNA}$ 基因 PCR 扩增的两个引物分别为 $27 \mathrm{~F}$ (正向 引物) : 5'-AGA GTT TGA TC(C/A) TGG CTC AG-3' (对应于 E. coli 16S rRNA 基因的第 8-27 个碱基位置) 和 1492R(反向引物) : 5'-GGT TAC CTT GTT ACG ACT T-3' (对应于 E. coli 16S rRNA 基因的第 $1492-1510$ 个碱基位置 $)^{[4]}$. 在 $20 \mu \mathrm{l}$ 反应体系中含有: 无菌蒸馏水 $14.4 \mu \mathrm{l}, 1 \times \mathrm{PCR}$ 缓冲液 $2 \mu \mathrm{l}, 1.5 \mathrm{~m} \mathrm{~mol} / \mathrm{L} \mathrm{MgCl}_{2}$ $1.6 \mu \mathrm{l}, 4 \times \mathrm{dNTP}$ 混合物 $0.4 \mu \mathrm{l}$,引物各 $0.2 \mu \mathrm{l}, 2.5 \mathrm{U} / \mu \mathrm{l}$ 的 Taq DNA 聚合酶 $0.2 \mu \mathrm{l}$, 模板 DNA $1 \mu \mathrm{l}$. PCR 反应 条件为: $96^{\circ} \mathrm{C}$ 预变性 $3 \mathrm{~min}$ 、接 $94^{\circ} \mathrm{C}$ 变性 $1 \mathrm{~min} 、 55^{\circ} \mathrm{C}$ 复性 $1 \mathrm{~min} 、 72^{\circ} \mathrm{C}$ 延伸 $2 \mathrm{~min}, 30$ 个循环后 $72^{\circ} \mathrm{C}$ 温育 $6 \mathrm{~min}$. PCR 扩增产物经 DNA 纯化系统( Wizard PCR Preps, Promega) 纯化后, 由上海博亚生物工程技术公司进行基 因序列测定.

（3）系统发育树的构建：将上述代表菌株的 $16 \mathrm{~S}$ rRNA 基因序列通过 NCBI 的 Blast 检索系统( http:// www. ncbi. nlm. nih. gov/Blast/) 进行序列同源性检索, 使用 ClustalX1.8 软件与从 GenBank 数据库中获得的 细菌 16S rRNA 序列进行多序列匹配排列 (Multiple Alignments), 采用邻接法 (neighbor joining method) 构建 系统发育树.

\section{3 菌种归类判定}

根据对细菌形态、理化特性的检验结果, 主要依据《Bergey's Manual of Determinative Bacteriology. 9th ed》、Austin B 等《Bacterial Fish Pathogens: Disease of Farmed and Wild Fish》及《Bergey's Manual of Systematic 
Bacteriology Volume 1 $\rangle^{[1,5,6]}$ 及有关资料, 并结合 $16 \mathrm{~S}$ rRNA 基因序列与系统发育学的结果, 进行供试菌的种 属判定. 另外, 择 1 个代表菌株送中国典型培养物保藏中心 (China Center for Type Culture Collection, CCTCC) 予以复核鉴定, 并做电子显微镜观察形态及鞭毛情况.

\section{4 人工感染试验}

择上述分离并经鉴定菌的代表菌株, 移接于普通营养肉汤管 $37^{\circ} \mathrm{C}$ 培养 $18 \mathrm{~h}$ 后, 制备成 $3 \times 10^{8} \mathrm{CFU} / \mathrm{ml}$ 的菌悬液, 分别经后肢基部接种感染健康成蟹 10 只 (每只剂量为 $0.1 \mathrm{ml}$ ), 同时设立接种同批、同剂量无菌 普通营养肉汤的对照蟹 10 只. 接种后隔离养殖于水簇箱中, 观察其发病及死亡情况, 对死亡蟹及时检查, 并按 1.1 及 1.2 中所述做细菌分离和对分离菌做相应的形态及理化特性等复核性检验. 以被感染蟹发病 或死亡并能重新分离到原感染菌为供试菌株的病原性判定指标, 对照蟹应在观察期内正常存活.

\section{5 药物敏感性测定}

随机择经鉴定后的纯培养菌株, 采用常规琼脂扩散 (K-B) 法进行对常用抗菌类药物的敏感性测定, 以 抑菌圈直径大小作为敏感与耐药的判定指标 ${ }^{[7]}$.

\section{2 结果}

\section{1 病变组织中的细菌}

上述 6 只病 (死) 蟹的肝胰腺中, 均有不同量的革兰氏染色阴性、杆状 (有的菌体稍弯曲)、两端钝圆、散 在(有的成双), 无芽孢, 大小多在 $(0.5-1.0) \mu \mathrm{m} \times(1.0-2.5) \mu \mathrm{m}$ (有的较大菌体长 $3.0 \mu \mathrm{m}$ 左右) 的 细菌.

\section{2 细菌分离与纯培养情况}

从 6 只蟹的肝胰腺中, 均分离到大量、在数量上差异不明显的 3 种细菌的菌落 (分别记作 $A 、 B 、 C$ ), 其 中的 A 菌即为本文所报告的鳗利斯顿氏菌. 其菌落特征为在普通营养琼脂上呈圆形光滑、边缘整齐、稍隆 起、浅橘黄色, 培养 $24 \mathrm{~h}$ 直径多在 $1.0 \mathrm{~mm}$ 左右 (半透明)、48 h 多在 $1.5 \mathrm{~mm}$ 左右 (不透明), 生长丰盛; 在血 液营养琼脂与普通营养琼脂培养基上的菌落特征基本一致, $\beta$-溶血(室温放置后更明显). 随机取每只蟹分 离菌落各 1 个移接于普通营养琼脂斜面做纯培养, 置 $4^{\circ} \mathrm{C}$ 保存供鉴定用; 如此 6 只蟹共做纯培养菌 6 株, 各 菌株按分离地、日期及株数依次编号为 HQ010516A-1 至 HQ010516A-6.

\section{3 分离菌的主要生物学性状及系统发育学情况}

2.3.1 形态特征 6 株纯培养菌的形态特征一致,均为革兰氏染色阴性、杆状及短杆状 (个别的近似球状) 、 一端或两端钝圆 (有的一端或两端稍尖)、多数菌体稍弯曲、散在、无芽狍、大小多在 $(0.3-0.5) \mu \mathrm{m} \times(0.7$ $-1.8) \mu \mathrm{m}$.

2.3 .2 不同培养基上的生长表现与菌落特征 6 株纯培养菌在供试同种培养基上的生长与菌落特征一致. 其在普通营养琼脂、血液营养琼脂上的与用此两种培养基从自然病(死)蟹肝胰腺中初代分离的相一致 ( $\beta$ 溶血为家兔血优于绵羊血), 在其他培养基上的生长及菌落特征如下.

(1) TSA：菌落呈圆形光滑、边缘整齐、稍隆起、不透明、橘黄色, 培养 $24 \mathrm{~h}$ 直径多在 $1.0 \mathrm{~mm}$ 左右、 $48 \mathrm{~h}$ 多在 $1.8 \mathrm{~mm}$ 左右,生长丰盛.

(2) TCBS: 培养 $24 \mathrm{~h}$ 检查未见细菌生长, $48 \mathrm{~h}$ 检查仅于划线接种起始部长出黄色菌苔及少数圆形光 滑、边缘整齐、稍隆起、直径在 $0.6 \mathrm{~mm}$ 左右的菌落, 刮下菌落(苔) 呈块状不易涂开,生长不良.

(3) XLD：形成圆形光滑、边缘整齐、较隆起、黄色的菌落, 培养 $24 \mathrm{~h}$ 直径多在 $0.5 \mathrm{~mm}$ 左右、48 h 多在 $1.5 \mathrm{~mm}$ 左右 (同心圆的脐状), 菌落边缘培养基下陷, 刮下菌落(苔) 呈黏块状不易涂开并留下黄色痕迹, 生 长中度.

(4) SS 琼脂: 形成圆形光滑、边缘整齐、较扁平、无色的菌落, 培养 $24 \mathrm{~h}$ 直径多在 $1.0 \mathrm{~mm}$ 左右、48 h 多在 $2.0 \mathrm{~mm}$ 左右, 孤立菌落中心为红点及形成围绕中心的花瓣状小再生菌落, 刮下菌落(苔) 呈黏块状不 易涂开并留下痕迹,生长较丰盛.

(5) 伊红美蓝琼脂: 仅于划线接种起始部长出圆形光滑、边缘整齐、稍隆起、同培养基本底色 (紫黑 色) 的小菌落, 培养 $24 \mathrm{~h}$ 直径多在 $0.2 \mathrm{~mm}$ 左右、 $48 \mathrm{~h}$ 多在 $0.4 \mathrm{~mm}$ 左右, 生长不良. 
(6) FA: 形成圆形光滑、边缘整齐、稍隆起、橘黄色的菌落, 培养 $24 \mathrm{~h}$ 直径多在 $0.6 \mathrm{~mm}$ 左右 (半透 明)、48 h 多在 $1.5 \mathrm{~mm}$ 左右(不透明), 生长较丰盛.

(7) 麦康凯琼脂: 形成圆形光滑、边缘整齐、稍隆起的无色菌落, 培养 $24 \mathrm{~h}$ 直径多在 $0.5 \mathrm{~mm}$ 左右、48 $\mathrm{h}$ 多在 $1.0 \mathrm{~mm}$ 左右 (菌落表面呈花纹状皱褶), 刮下菌落(苔) 呈黏块状不易涂开并留下痕迹, 生长中度.

(8) RS：呈圆形光滑、边缘整齐、稍隆起的黄色菌落 (菌落边缘处下陷), 培养 $24 \mathrm{~h}$ 直径多在 $0.8 \mathrm{~mm}$ 左右、48 h 多在 $1.5 \mathrm{~mm}$ 左右, 刮下菌落呈块状不易涂开并留下黄色痕迹, 生长中度.

(9) $2216 \mathrm{E}$ ：形成圆形光滑、边缘整齐、稍隆起、半透明、浅橘黄色的菌落, 培养 $24 \mathrm{~h}$ 直径在 $0.5 \mathrm{~mm}$ 左 右、48 h 多在 $1.5 \mathrm{~mm}$ 左右, 生长中度.

(10) 庆大霓素琼脂: 仅于划线接种起始部位长出菌苔及圆形光滑、边缘整齐、稍隆起的浅灰褐色菌 落, 培养 $24 \mathrm{~h}$ 直径多在 $0.8 \mathrm{~mm}$ 左右、48 h 多在 $2.0 \mathrm{~mm}$ 左右, 虽生长的菌落(苔) 较丰盛但仅于接种起始部 位生长, 难以在连续划线处长出单菌落.

2.3 .3 不同培养温度及在普通营养肉汤中的生长情况 6 株供试菌在 $28^{\circ} \mathrm{C} 、 37^{\circ} \mathrm{C}$ 条件下生长情况基本一致 $\left(28^{\circ} \mathrm{C}\right.$ 的更优些), 菌苔不透明、橘黄色,生长丰盛. 在普通营养肉汤中, 6 株菌生长表现一致,均呈均匀混浊 生长, 管底形成小圆点状菌体沉淀 (摇动后即消散), 有较厚的菌膜形成 (摇动后呈较大的块状悬浮不易散 开并留下明显菌环).

2.3.4 理化特性 6 株菌对所测项目结果一致,具体如表 1 所示.

表 1 分离菌的理化特性 ${ }^{1)}$

Tab. 1 Physiological and biochemical characteristics of isolates

\begin{tabular}{|c|c|c|c|c|c|}
\hline 项 目 & 果 & 项 & 结 & 项 目 & 结 \\
\hline $37^{\circ} \mathrm{C}$ 生长 & + & 蔗糖 & + & 麦芽糖 & + \\
\hline 氧化酶 & + & 鼠李糖 & - & 丙二酸盐利用 & - \\
\hline 接触酶 & + & 肌醇 & - & 醋酸盐利用 & - \\
\hline 产 $\mathrm{H}_{2} \mathrm{~S}$ & - & 甲基红 & + & 木糖 & - \\
\hline $\mathrm{O}-\mathrm{F}$ 试验 (葡萄糖) & $\mathrm{F}$ & V-P 试验 & + & 木糖醇 & - \\
\hline 动力 (半固体) & + & 阿拉伯糖 & - & 苦杏仁苷 & - \\
\hline 明胶液化 & + & 甘露醇 & + & 松三糖 & - \\
\hline 柠檬酸盐利用(Simmons) & + & 吲哚 & + & 山梨糖 & - \\
\hline 葡萄糖:产酸 & + & 七叶苷利用 & - & 侧金戞花醇 & - \\
\hline 产气 & - & 苯丙氨酸脱氨酶 & - & 酒石酸盐利用 & - \\
\hline 山梨醇 & + & 尿素酶 & - & 黏液酸利用 & - \\
\hline 蜜二糖 & - & IPA ( SIM) & - & $\beta$-半乳糖苷酶 & + \\
\hline 棉子糖 & - & 阿拉伯醇 & - & $\alpha$-甲基-D-葡糖苷 & - \\
\hline 菊糖 & - & 乙酰胺酶 & - & 乳糖 & + \\
\hline 水杨苷 & - & 淀粉酶 & + & 海藻糖 & + \\
\hline 吐温 80 & + & 糊精 & + & 赤藓醇 & - \\
\hline 硝酸盐还原 & + & 卫茅醇 & - & 甘露糖 & + \\
\hline 半乳糖 & + & 甘油 & + & 果糖 & + \\
\hline 纤维二糖 & + & $\mathrm{NaCl}$ 中生长 & - & 松二糖 & - \\
\hline 蛋白酶 & + & $1 \%$ & + & $\mathrm{O} / 129: 10 \mu \mathrm{g}$ & $\mathrm{S}$ \\
\hline DNA 酶 & + & $6 \%$ & - & $150 \mu \mathrm{g}$ & $\mathrm{S}$ \\
\hline 卵磷脂酶 & - & & & & \\
\hline
\end{tabular}

1) + 示阳性, - 示阴性, F 示发酵型, $\mathrm{S}$ 示敏感.

2.3.5 16S rRNA 基因序列和系统发育学 代表菌株(HQ010516A-1 株) 在 GenBank 中登录号为 DQ068758, 所扩增的 $16 \mathrm{~S}$ rRNA 基因序列长度为 $1423 \mathrm{bp}$, 将该菌株的 $16 \mathrm{~S} \mathrm{rRNA}$ 基因序列通过 NCBI 的 Blast 进行同源 性检索,结果其与弧菌属细菌的 $16 \mathrm{~S}$ rRNA 基因序列自然聚类, 取 25 株弧菌属细菌的 $16 \mathrm{~S} \mathrm{rRNA}$ 基因序列与 其进行系统发育学分析,结果该菌株与鳗利斯顿氏菌 (含原鳗弧菌) 和奥氏弧菌 (Vibrio ordalii; 该菌即原鳗 
弧菌生物型 II ) 聚为一族, 其系统发育树如图 1 所示.

\section{4 细菌种类}

上述检验结果表明, 所分离鉴定的 6 株 菌为同种细菌, 依据其形态特征、理化特性及 系统发育学等, 判定为利斯顿氏菌属的鳗利 斯顿氏菌( 即原弧菌属的鳗弧菌). 经择 HQ010516A-1 株送 CCTCC 做复核鉴定, 结果 同样判定为鳗利斯顿氏菌, 电镜观察其菌体 稍弯曲、端生单鞭毛.

\section{5 人工感染的致病作用}

择经鉴定的鳗利斯顿氏菌 HQ010516A-1 株,按 1.4 中所述方法做对中华线鳌蟹的感 染试验, 结果 10 只蟹于感染后 $48 \mathrm{~h}$ 内死亡, 死亡蟹均无明显眼观病变; 对照蟹观察 $10 \mathrm{~d}$ 均正常存活.

随机取 3 只感染死亡蟹的肝胰腺, 直接 做抹片经革兰氏染色镜检, 结果发现有大量 在形态特征上与感染菌相同的革兰氏染色阴 性细菌. 同时, 做细菌分离培养均分离到大量 生长、纯一的同原感染菌的菌落, 取从每只蟹 分离的各 1 个菌落做纯培养共 3 株, 分别进 行形态特征及理化性状的复核鉴定, 结果与 原感染菌一致.

\section{6 药物敏感性}

随机取鳗利斯顿氏菌 3 株 (HQ010516A1、HQ010516A-2、HQ010516A-3), 做对 37 种 抗菌类药物的敏感性测定. 结果均表现对供 试的头孢噻朊、头孢曲松、头孢他啶、头孢哌 酮、头孢吡肜、氨曲南、阿奇雼素、链雨素、卡 那需素、庆大需素、妥布雼素、丁胺卡那霉素、 新震素、大观雼素、诺氟沙星、氧氟沙星、环丙 沙星、氯霉素、恩诺沙星、复方新诺明、甲氧苄
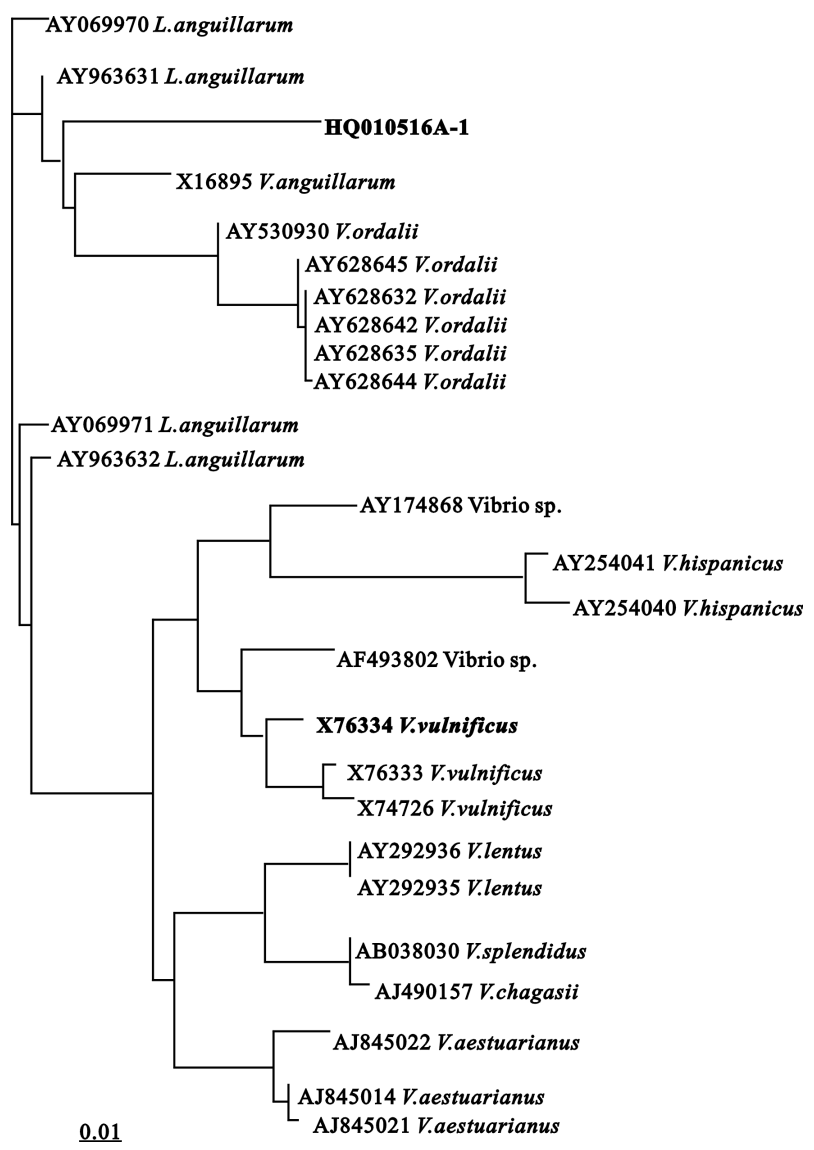

图 $116 \mathrm{~S}$ rRNA 基因序列分析聚类图 ( 图中 AY069970 - AJ845021 为菌株 在 NCBI 的 $16 \mathrm{~S}$ rRNA 基因登录号)

Fig. 1 Dendrogram of 16S rRNA gene sequences analysis ( AY069970 - AJ845021 were database accession numbersof 16S rRNA gene in NCBI)

啶、呋喃妥因、呋喃唑酮、四环素、多西需素、多黏菌素 B、利福平、新生霨素、红需素等 29 种药物呈现高度敏 感或敏感 (抑菌圈直径在 16-40 mm); 对头狍唑啉、克林需素、万古霉素 3 种药物低度敏感 (抑菌圈直径在

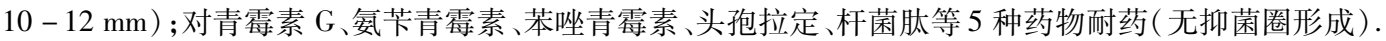

\section{3 讨论}

近年来,由某些病原细菌所致的中华线鳌蟹病害在国内外已多有记载和报道. 如国内顾志峰等报道由 类立克次氏体 (Richettsia-Like organisms)引起的“颤抖病” 及病理变化 ${ }^{[8,9]}$; 沈锦玉、陆宏达等从患“腹水病” 及“抖抖病”并发病的中华线鳌蟹中, 分离到嗜水气单胞菌 (Aeromonas hydrophila) 和拟态弧菌 (Vibrio mimicus $)^{[10,11]}$; 李文宽等从中华线螯蟹体内分离到副溶血弧菌 $(V \text {. parahaemolyticus })^{[12]}$; 徐海圣等分离到豚鼠气 单胞菌 (A. caviae) 和易损气单胞菌 $(A . \text { trota })^{[13,14]}$; 李华等曾分离到弗氏柠檬酸杆菌 ${ }^{[15]}$, 通过人工感染试验 表明均可导致健康蟹的发病与死亡; 朱越雄等曾分离到类志贺邻单胞菌 (Aeromonas shigelloides) ${ }^{[16]}$,但经 对健康蟹的感染试验未见发病; 早在 20 世纪 70 年代, Sizemore、Phillips 及 Fishbein 等即从篮蟹 (blue crab) 
体内检出副溶血弧菌等多种细菌 ${ }^{[17-19]}$. 本次所检中华线鳌蟹病例, 尽管是由鳗利斯顿氏菌、维氏气单胞菌 和弗氏柠檬酸杆菌 3 种细菌引起的混合感染, 但经对健康蟹所做人工感染试验, 表明所检出的撸利斯顿氏 菌具有较强的致病作用,也进一步表明了该菌在水产养殖动物中的广泛致病性.

通过用普通营养琼脂等 12 种不同培养基做划线接种后 $28^{\circ} \mathrm{C}$ 培养观察, 发现该菌在供试的普通营养琼 脂、血液(家兔血或绵羊血) 营养琼脂、TSA、FA、XLD、RS、SS 琼脂、庆大霉素琼脂、麦康凯琼脂、2216E 等 10 种培养基上生长均较好; 在 TCBS、伊红美蓝琼脂培养基上生长不良. 因此认为对该菌分离鉴定时, 可根据 其在这些供试培养基上的生长情况予以选择使用.

经以 37 种抗菌类药物对 3 株鳗利斯顿氏菌的敏感性测定,尚未发现在不同菌株间对某种抗菌类药物 敏感或耐药的明显差异性, 这一结果初步显示所测菌株在对供试抗菌类药物中所表现出的一致性, 有益于 对选择用药防治时的参考.

\section{4 参考文献}

[1] Austin B, Austin D A. Bacterial Fish Pathogens: Disease of Farmed and Wild Fish. Third( Revised) Edition. Chichester Praxis Publishing Ltd,1999:29 - 32,106 - 140.

[2] 吴后波,潘金培. 弧菌属细菌及其所致海水养殖动物疾病. 中国水产科学,2001,8(1):89-93.

[3] 冬秀珠,蔡妙英. 常见细菌系统鉴定手册. 北京:科学出版社, 2001,353-398.

[4] Martin F Polz, Collen M Cavanaugh. Bias in template to product ratios in multitemplate PCR. Appl Environ Microbiol, 1998,64 (10):3724-3730.

[5] Holt J G, Krieg N R, Sneath P H A, et al. Bergey's Manual of Determinative Bacteriology, Ninth Edition. Baltimore: Williams and Wilkins, 1994:190 - 194,253 - 274.

[6] Krieg N R, Holt J G. Bergey's Manual of Systematic Bacteriology. Volume 1. London: Williams and Wilkins, Baltimore, 1984:518-538.

[7] 叶应妩,王毓三. 全国临床检验操作规程 (第二版). 南京: 东南大学出版社, 1997:553-560.

[8] 顾志锋,王 文,杜开和等. 患 “颤抖病” 中华线鳌蟹体内类立克次氏体生物的寄生. 湖泊科学, 2000, 12(3) :289-291.

[9] 顾志锋,王 文,杜开和等. 中华线鳌蟹体 “㗜抖病”病原、病理学初步研究. 湖泊科学, 2000,12(4): $367-371$.

[10] 沈锦玉,尹文林,钱 冬等. 中华线螯蟹“腹水病”及 “抖抖病” 并发病病原的研究. 中国水产科学, $2000,7(3): 89-92$.

[11] 陆宏达,金丽华,范丽萍等. 中华线鳌蟹细菌性病原的分离和鉴定. 水产学报, 1999,23(4):381-386.

[12] 李文宽,于 翔,闻秀荣等. 辽宁地区河蟹暴发性流行病病原研究. 水产科学, 1999,18(4):10-13.

[13] 徐海圣,徐步进. 中华线鳌蟹细菌性病原的分离与鉴定. 中国兽医学报,2002,22(3): 137-139.

[14] 徐海圣, 黄立锋, 王淑霞. 中华线鳌蟹豚鼠气单胞菌的分离和鉴定. 浙江大学学报 (农业与生命科学 版) ,2001,27(6):677-681.

[15] 李 华,刑殿楼,白国福等. 弗氏柠檬酸杆菌对河蟹致病性的研究. 水生生物学报. 2001,25(3):217 -223 .

[16] 朱越雄, 巩成良,薛仁宇等. 中华线鳌蟹组织中一株类志贺邻单胞菌的分离与特性分析. 中国微生态学 杂志, $2001,13(5): 263-264$.

[17] Sizemore R K, Colwell R R, Tubiash HS, et al. Bacterial flora of the hemolymph of the blue crab, Callinectes sapidus: Numerical taxonomy. Applied Microbiology, 1975,29(3):393 - 399.

[18] Phillips F A, Peeler J T. Bacteriological survey of the blue crab industry. Applied Microbiology, 1972, 24 (6) :958 - 966.

[19] Fishbein M, Melilman I J, Pitcher J. Isolation of V.parahaemolyticus from the processed meat of Chesapeake Bay blue crab. Applied Microbiology,1970,20:176 - 178. 